\title{
The corneal confocal microscopy today
}

\author{
Cagini $\mathrm{C}^{\mathbf{1}}$, Torroni $\mathrm{G}^{\mathbf{1}}$ and Messina $\mathrm{M}^{\mathbf{1}}$ \\ ${ }^{1}$ University of Perugia, Department of Surgical and Biomedical Sciences, Division of Ophthalmology, Italy
}

The extreme transparence of the corneal structure, which reflects only $1 \%$ of the incident light, makes its observation arduous. Over the last 30 years, several techniques have been proposed for the macroscopic and microscopic corneal examination and among them a key role is represented by the confocal microscopy. Nowadays this non-invasive and painless technique has allowed histological studies of the human corneal microstructure both in healthy and pathological condition. The term "confocal" describes the feature of this microscope: the observation point and the illumination source converge in a common point, "common focal point". This permits that the reflections of the light from the areas close to the observation point, "chatter" in microscopy, are blocked with an increase in resolution and being consequently able to work at higher magnification. Compared to the simple microscopy the quality of the image is then improved because the images coming from multiple depths of the sample are not overlapped. In other words, while a conventional microscope "sees" the sample till the light can penetrate, the confocal microscope examines from time to time only the images of a specific depth level. Layer by layer, the information related to the corneal alterations are equal to the ones obtainable with cytological and histological exams. This technique is nowadays widely used in numerous biological sciences, from biology to genetic and for the study of corneal layers in ophthalmology. The maximum resolution of the confocal microscopy is very high, around 5-6 $\mu \mathrm{m}$ or the red globule dimension. The corneal cells, their nuclei and cytoplasm (but not their organelles) and some corneal pathogenic agents can then be seen by this microscope. The corneal confocal microscopy allows qualitative and quantitative corneal analysis. The qualitative ones offer different possibilities for each corneal layer: the morphology and integrity of the corneal epithelial cells can be evaluated; at the level of the sub basal plexus the whole nerves architecture can be studied allowing a detailed evaluation of their tortuosity, morphology and reflectivity; the stromal layer and the cellular activity can be assessed as an inflammatory marker of the stromal keratocytes and lastly, at the endothelial level, it allows a detailed evaluation of its integrity and mainly the evolution of the morphology of the cells, which normally is hexagonal. The quantitative analysis can be carried out either with an internal or an external software (Image J) and allows the corneal thickness measurement, the depth of a lesion, the calculation of the number of nerve fibers in a specific area (ROI), the calculation of the number of corneal nerve fibers, the calculation of the nerve fibers angulation (objective sign of tortuosity) and finally the dimensional measurement of a noxious substance.

At the moment, the confocal microscopy is applied with success in the corneal field in 4 main areas: the in vivo characterisation of corneal degenerations linked to different noxious substances, the corneal dystrophies, the differential diagnosis in the presence of keratitis or keratopathies and lastly the microstructural analysis of corneas underwent surgery. For instance, any corneal dystrophy, which is today still clinically diagnosed, has a peculiar confocal aspect which allows in specific cases to rectify a wrong clinical interpretation or to directly diagnose a specific dystrophy. A clear example is the Fuch's endothelial dystrophy in which, in case of stromal corneal oedema, the opacity does not permit a detailed examination of the endothelium, even using a conventional microscopy. In this case, the only instrument that allows a direct evaluation of the endothelial layer and an exact calculation of its thickness and number of healthy cells, is the confocal microscope. It has a key role in surgical situations.

The specific signs of some epithelial dystrophies, such as Cogan and Meesmann's dystrophy are clearly seen with this instrument and an accurate differential diagnosis can be made between some stromal dystrophies, being the microscope able to identify and differentiate, like in the type I or II Reis Buckler's dystrophy and in the type I and II Granular dystrophy, the deposit type and its morphology and distribution. Even in the presence of infective keratitis it is possible to discriminate between the different cellular components like the inflammatory cells and, in some cases, the micro-organism causing the infective process. The dimension of the vast majority of micro-organisms is about $1-3 \mu \mathrm{m}$ and they cannot be seen with the instrument; however, the confocal microscopy can detect indirect signs of infection or inflammation such as oedema or leucocytes. When the Acanthamoeba parasite is involved, the confocal microscope allows the direct visualisation of the cysts and the trophozoites resulting highly useful in the diagnosis and treatment of this infection. Although the confocal microscopy is not the gold standard in diagnosing the Acanthamoeba keratitis, it is without any doubt a valid option for detecting the infection and cut diagnosis time making it possible to begin the right treatment.

The confocal microscopy is also important to identify the peculiar findings of the fungal infections such as hyphae, spores, arthroconidia and pseudohyphae and often hypothesise, with the quantitative analysis, which fungus is involved. In fact, some software allows the analysis of the involved fungal micro-structures providing an initial microorganism characterisation.

Moreover, over the last years special attention is given to the corneal immune response in infective and inflammatory cases, trying to differentiate the subtypes of typical leucocytes of specific clinical pictures to identify, based on the immune response seen, the type of infection and the inflammatory state, acute or chronic. As a matter of fact, using the confocal microscopy we can distinguish between leucocytes and lymphocytes: the former is a clear sign of an immune

Correspondence to: Cagini C, University of Perugia, Department of Surgical and Biomedical Sciences, Division of Ophthalmology, Ospedale S. Maria della Misericordia,S. Andrea delle Fratte, Perugia, Italy, Tel: +39 75 5783944; Fax: +39 75 5783951; E-mail: carlo.cagini@unipg.it , carlocagini@hotmail.com

Received: May 2, 2017; Accepted: June 06, 2017; Published: June 09, 2017 
response supported by leucocytes which are typically seen in acute inflammatory states while the latter characterise the chronic one.

It is also possible to identify the corneal leucocytes (Langerhans or antigen presenting cells) which are an obvious sign of a latent inflammatory state even if seen in apparently quiet corneas as can be seen in the untreated eye dry disease.

Having the confocal microscopy the quality to acquiring corneal images in four dimensions $(\mathrm{x}, \mathrm{y}, \mathrm{z}, \mathrm{t})$ at the cellular level, it results suitable to monitor the epithelial and stromal wound healing process, mainly following surgical procedures. It also allows, in case of refractive surgery, the measurements of the marginal wound space, of the epithelial regrowth depth and of the corneal fibrosis grade. Similar evaluations can be carried out also following corneal collagen cross linking, penetrating keratoplasty, anterior and posterior lamellar keratoplasties (DALK, DSAEK, DSEK, DMEK, PDEK).

In other cases, it represents a useful instrument to demonstrate inter or intra-cellular pathological depositions in the different corneal layers seen in corneal manifestations of metabolic diseases.

To sum up, nowadays using the confocal microscopy during inflammatory states, wound healing processes, toxicity and infections, it is possible to dynamically study over time corneal cellular details and to follow the evolution, the treatment response and eventually the microscopic complications.

\section{References}

1. Smedowski A, Tarnawska D, Orski M (2017) Cytoarchitecture of epithelial inflammatory infiltration indicates the aetiology of infectious keratitis. Acta Ophthalmol . [Crossref]

2. Maycock NJ, Jayaswal R (2016) Update on Acanthamoeba Keratitis: Diagnosis, Treatment, and Outcomes. Cornea 35: 713-720. [Crossref]

3. Weiss JS, Møller HU, Aldave AJ, Seitz B, Bredrup C, et al. (2015) IC3D classification of corneal dystrophies--edition 2. Cornea 34: 117-159. [Crossref]

4. Iaccheri B, Torroni G, Cagini C (2017) Corneal confocal scanning laser microscopy in patients with dry eye disease treated with topical cyclosporine. Eye: 788-794. [Crossref]

5. Kheirkhah A, Syed ZA, Satitpitakul V (2017) Sensitivity and Specificity of Laserscanning In Vivo Confocal Microscopy for Filamentous Fungal Keratitis: Role of Observer Experience. Am J Ophthalmol Apr 23. [Crossref]

6. You JY, Botelho PJ (2016) Corneal In Vivo Confocal Microscopy: Clinical Applications. R I Med J (2013) 99: 30-33. [Crossref]

7. Cruzat A, Qazi Y, Hamrah P (2017) In Vivo Confocal Microscopy of Corneal Nerves in Health and Disease. Ocul Surf 15: 15-47. [Crossref]

Copyright: (C2017 Cagini C. This is an open-access article distributed under the terms of the Creative Commons Attribution License, which permits unrestricted use, distribution, and reproduction in any medium, provided the original author and source are credited. 